Research Article

\title{
Clinical features of hospitalized dengue patients in Sri Lanka from 2004 to 2006
}

\author{
Messer WB, ${ }^{1,2}$ Kanakaratne N, ${ }^{3}$ Thevanesam V, ${ }^{4}$ Ranawaka G, ${ }^{3,5}$ Shahani A, ${ }^{6}$ de Silva AM, ${ }^{2}$ \\ Gunasekera M. . $^{*}$
}

Sri Lanka Journal of Infectious Diseases 2012 Vol.1(2);9-18

DOI: http://dx.doi.org/10.4038/sljid.v2i1.3004

Key words: Dengue Fever; clinical features

\begin{abstract}
The recent emergence of dengue hemorrhagic fever (DHF) in Sri Lanka has been well documented. Here, we present the results of clinical and laboratory evaluation of adult and pediatric PCR positive dengue patients who were hospitalized in Sri Lanka during 2004-2006. Three hundred and fifty seven patients were included in the study. Sixty five patients were pediatric (aged 0-12) and 292 patients were adult (aged 13-80). Patients were classified as having dengue fever (DF), dengue fever with hemorrhagic signs (DFHem) and dengue hemorrhagic fever (DHF). Clinical manifestation of disease differed qualitatively between children and adults. Vomiting and flushing were most common in children and arthralgia/myalgia were the most common finding in adults. Thrombocytopenia was more common in adults than in children. A subgroup of 185 patients had the infecting serotype identified by PCR. In the pediatric group, three serotypes, DENV2, DENV3, and DENV4 were found. DENV3 was the most common overall and in all pediatric diagnoses. DENV3 and DENV2 were encountered in pediatric DHF patients but not out of proportion to their

\footnotetext{
${ }^{1}$ Department of Medicine, Division of Infectious Diseases, University of North Carolina School of Medicine, Chapel Hill, NC

${ }^{2}$ Department of Microbiology and Immunology, University of North Carolina, Chapel Hill, North Carolina

${ }^{3}$ Genetech Research Institute, Colombo, Sri Lanka

${ }^{4}$ Department of Microbiology, Faculty of Medicine, University of Peradeniya, Sri Lanka.

${ }^{5}$ Department of Zoology, Faculty of Natural Sciences, Open University of Sri Lanka.

${ }^{6}$ Apollo Hospital, Colombo, Sri Lanka

*Deceased
}

Address for correspondence: Dr. W.B.Messer, Fellow, UNC School of Medicine, Dept. of Medicine, Div. of Infectious Diseases, CB\# 7030, 130 Mason Farm Road, 2nd Floor Bioinformatics, Chapel Hill, NC 27599. Email: wmesser@unch.unc.edu Tel: (919) 966-2536:Fax: (919) 966-6714 
representation in all pediatric patients. All four serotypes were identified in the adult group, with DENV3 and DENV2 being the most common. All four serotypes were found in DHF patients, with DENV3 occurring most frequently but not out of proportion to its occurrence in all adult patients. Our findings, combined with other recent reports of DENV in Sri Lanka, should help us better understand the evolving picture of dengue disease in Sri Lanka.

\section{Introduction}

Dengue virus (DENV) infects millions of people throughout the tropics and subtropics each year. Symptomatic dengue infection most often presents as Dengue Fever (DF), but tens of thousands of these infections progress to a life threatening disease known as dengue hemorrhagic fever (DHF). ${ }^{1}$ DENV occurs primarily in urban settings that support large populations of the mosquito vector. Despite impressive advances in the prevention and control of some infectious diseases, dengue has so far evaded efforts at prevention and control. Currently there are no anti-viral medications or approved vaccines against DENV., 3

Dengue is a positive stranded RNA virus belonging to the Family Flaviviridae and Genus Flavivirus. There are 4 serotypes designated DENV1-4. Infection with one serotype is believed to provide long-term immunity to that serotype, but not to the other serotypes. Thus, people can be infected with multiple serotypes during their lifetime. ${ }^{4}$ Some people infected with DENV are asymptomatic, whereas others develop disease, including DHF, which can be fatal. Pre-existing immunity is a risk factor for severe disease, as multiple studies have shown that individuals with secondary dengue infections have a greater risk of developing DHF. ${ }^{4} 5$ Furthermore, all four serotypes of DENV can cause DHF, but within each serotype some genotypes are linked to severe disease and others to mild disease. ${ }^{6}$

In the last 30 years DENV has spread to new regions worldwide and there has been an increase in disease incidence and severity in areas where the virus has long been in circulation. The epidemiology of dengue in the island of Sri Lanka is particularly interesting as before 1989, despite co-circulation of all 4 serotypes and many repeat infections in the population, there were very few patients with DHF. In 1989, the incidence of DHF increased dramatically and large numbers of DHF cases have been documented every year after 1989. ${ }^{7}$ The number of DF and DHF cases in Sri Lanka has continued to increase and two of the largest epidemics occurred in 2002 and 2004. ${ }^{8}$ Genetech Molecular Diagnostics Laboratory in Sri Lanka has been offering a reverse transcriptase PCR (RT-PCR) test to detect DENV in clinical specimens. Using this, the molecular epidemiology of DENV in Sri Lanka from 2003 to 2006 has been previously

described. ${ }^{8}$ The current study describes in detail the clinical and laboratory findings from patients hospitalized with dengue fever in Colombo over the same period.

\section{Materials and methods}

Study Population and patient selection: Sri Lanka is an island nation off the southeastern coast of India. It had an estimated population of 19,886,000 in 2006. The majority of patients in this study are from Colombo District, a largely urban area which had an estimated population of $2,421,000$ in 2006. The age structure in the Colombo district is that of a developing world urban 
center with over $50 \%$ of the population aged 29 yrs or less. Subjects were patients admitted to Apollo, Nawaloka and Durdans Hospitals in Colombo from March, 2004 to January, 2006. Serum samples collected from pediatric and adult patients in these hospitals with fever for 4 or fewer days and suspected dengue infection were tested for evidence of active DENV infection by Genetech Research Institute using methods described below. Chart review was subsequently performed on available DENV PCR positive patients. The study was reviewed and approved by the Institutional Review Boards of the University of North Carolina at Chapel Hill, USA and the Ethical Committee, Faculty of Medicine, University of Peradeniya, Sri Lanka.

Definitions: WHO classification for dengue disease were used except as outlined below. DF was defined as fever, PCR positive for DENV and no evidence of bleeding. DF with unusual hemorrhage (DFhem) was defined after Harris ${ }^{9}$ as DF with evidence of bleeding (petechiae, purpura, melena, hematemesis, epistaxis, gingival bleeding, or hematuria), but without thrombocytopenia. The definition of DHF was modified from the commonly referenced World Health Organization (WHO) definition. ${ }^{1}$ The WHO has established a standardized classification system for DHF which requires evidence of thrombocytopenia $\leq 100,000$, haemoconcentration $>20 \%$ of the upper limit of normal for the patient age, sex and population and a positive tourniquet test. As previously noted ${ }^{10,11}$, proper medical management of a patient with suspected DHF includes intravenous fluid resuscitation that causes haemodilution, which modifies the haemoconcentration observed in DHF. Moreover, the tourniquet test is a relatively time consuming, poorly defined and uncomfortable procedure for the patient. Hence the WHO classification system is difficult to apply in hospitalized patients because proper patient care eliminates haemoconcentration and clinicians infrequently use the tourniquet test in the inpatient setting. Consequently, these 2 criteria were not included in the definition used in the current study and DHF was defined as DF with thrombocytopenia $\leq 100,000 \mathrm{~mm}^{3}$ and evidence of bleeding (petechiae, purpura, melena, hematemesis, epistaxis, gingival bleeding, or hematuria).

Laboratory Methods: Reverse-Transcription Polymerase Chain Reaction (RT-PCR) was performed using the DV1 and DV3 primer set and the ALD 1 and ALD 2 primer set in a single reaction $^{12}$. The DV primers amplify a 470 base pair fragment from the NS3 gene of all Flaviviruses. The ALD1 and ALD2 primers amplify a 229-240 base pair product from the 3' untranslated region (UTR) of all DENVs. Nested PCR was subsequently performed using the DV amplified sequences to serotype the viruses.

Statistical Analysis: All data were entered into and analyzed using JMP statistical software ${ }^{13}$. Student's T-test was used to compare means and the chi-square test was used to compare all count data. In all cases observed statistical differences were considered significant for $\mathrm{P} \leq 0.05$.

\section{Results}

Demographic Data: A total of 523 PCR positive patients were detected between 3/2/2003 and $1 / 8 / 2006$. Of those, 357 had the necessary clinical and laboratory data for disease classification using the definitions described above. Patient ages ranged from 0 to 80 with a mean age of 28.74 and a male to female ratio of 206: 151 (Table 1). The overall ratio of males to females is different from the male to female ratio in the Colombo District population ${ }^{14}$. The mean ages of males and females however, were not significantly different (T-test, $\mathrm{P}>0.05$ ). The age distribution of cases was distinctly bimodal, with two peaks observed, one at $<1$ yrs old and one 
at 32 yrs old. The median and mean ages of pediatric ${ }^{15}$ (0-12 years old) and adult patients 16,17 (13-85 years old), were 4.77 and 3 years and 34.08 and 32 years respectively (Table 1). Males outnumbered females in both groups, (Table 1) though the differences were not statistically significance for either group (Chi-square test, $\mathrm{P}>0.05$ ). The mean ages of males and females were not significantly different (T-test, $\mathrm{P}>0.05)$.

Diagnoses: For all patients, the most frequently diagnosed condition was DF, accounting for over three-quarters of all diagnoses (Table 2). DFHem was the least likely diagnosis, accounting for only $3.92 \%$ of all patients, while $16.25 \%$ of the patients met the criteria for DHF (Table 2). There was no statistically significant difference in risk of disease by sex (Chi-square test, $\mathrm{P}>0.05)$.

A similar pattern was seen in both pediatric and adult patients. DF (84.62\%) accounted for the vast majority of pediatric patients, DHF for $9.23 \%$ and DFHem for $6.15 \%$ (Table 2). Among children, there was no observed difference between males and females in any diagnostic category. Among adults, DF was diagnosed in $78.77 \%$, Although a higher percentage of adults appeared to present with DHF - $17.81 \%$ vs $9.23 \%$ in children (Table 2), the observed difference was not statistically significant (Chi-square test, $\mathrm{P}>0.05$ ). As seen with pediatric patients, there were no statistically significant differences between males and females and the likelihood of any diagnostic category (Chi-square test, $\mathrm{P}>0.05$ ). Overall, children were no more or less likely to develop DF, DFHem or DHF when compared to adults.

Clinical Findings: A wide variety of symptoms and signs were elicited. While several complaints were equally common in children and adults, the two groups had some notable differences. The dominant complaints in pediatric patients were vomiting (49\%), flushing (48\%) and abdominal pain (29\%) (Figure 1). Although these 3 complaints were seen in adults at similar frequency $(41 \%, 57 \%$ and $30 \%$ respectively) the dominant complaints in adult patients were myalgia/arthralgia (64\%), followed by headache (59\%) (Figure 1). In contrast, myalgia/arthralgia and headache were relatively rare complaints in children ( $8 \%$ and $14 \%$ respectively).

Clinical findings were further grouped by the study diagnostic criteria within age groups (Figures $2 \mathrm{a}$ and $2 \mathrm{~b}$ ). In children, there were three findings that appeared qualitatively more closely associated with certain disease states. Flushing was a much more common complaint among pediatric DHF and DFHem patients (83\% and $100 \%$ respectively) and rare in pediatric DF patients (40\%). Abdominal pain was also a more common complaint among children with bleeding (50\% and $100 \%$ in DFHem and DHF respectively) while only $20 \%$ of DF patients complained of abdominal pain. Finally, hepatomegaly was a more common finding for pediatric DHF patients, $67 \%$ compared to $15 \%$ of DF patients and $25 \%$ of DFHem patients. While hepatomegaly was detected with greater frequency in the pediatric patients $(20 \%$ vs $5 \%$ in adults), this may be due more to ease of detection in pediatric cases because of body habitus. No comparative statistics were done given the large number of groups and limited absolute numbers within many of these groups.

In adults, the frequency of complaints was generally similar in patients with DF and DFHem with the exception of flushing (Figure 2b). Abdominal pain and loose stools were also less prominent complaints in patients with DF compared to those with DFHem or DHF (Figure 2b). 
As with pediatric patients, no statistical tests were performed on these comparisons since the absolute numbers were small and number of categories were high.

Laboratory findings: By definitions used in the study, DFHem and DHF patients differed by platelet count - DFHem patients did not have thrombocytopenia (platelets $>100,000$ ) and DHF patients had thrombocytopenia. Thrombocytopenia was present in 58.7\% ( $=158)$ of adult patients while it was present in only $30.9 \%(\mathrm{~N}=17)$ of pediatric patients. These proportions differed significantly (chi-square test $\mathrm{p} \leq 0.05$ ) from expected proportions.

Serotyping data was available for 185 patients in the study. DENV3 was the most frequently identified serotype $(61.54 \%)$ in paediatric patients. with DENV2 in $30.77 \%$ and DENV4 in $7.69 \%$ of the patients (Table 3). DENV1 was not detected in any of the pediatric patients. DENV3 was identified most frequently in all diagnostic groups accounting for $66.67 \%$ of DHF patients, $100 \%$ of DFHem patients and $59.09 \%$ of DF patients. DENV2 was identified in approximately one-third of DHF $(33.33 \%)$ and DF patients $(31.25 \%)$, while DENV4 was only present in DF patients (12.50\%) (Table 3). While DENV3 was found most frequently in DHF patients, its frequency was not out of proportion to its occurrence in all patients (Chi-Square test, $\mathrm{P}>0.05)$.

For adult patients, a total of 159 samples were serotyped. As with the pediatric patients, DENV3 was identified most frequently, accounting for $51.57 \%$ of all patients, followed by DENV2 at $37.11 \%$. DENV1 and DENV4 were both present but much less common at $6.26 \%$ and $5.03 \%$ respective. Stratification of serotype by diagnostic group yielded results similar to those observed in pediatric patients. DENV3 was the serotype found most frequently in all diagnostic categories, accounting for $51.57 \%$ of DHF patients, $75 \%$ of DFHem patients and $50.39 \%$ of DF patients (Table 3). DENV2 was the second most frequently identified serotype in all diagnostic categories followed by DENV1 and DENV4 (Table 3). All four serotypes were identified in DHF patients. While DENV3 was found more frequently than all other serotypes in DHF patients, as with the pediatric patients, the proportion of DENV3 positive DHF patients did not differ from the proportion of DENV3 positive patients overall, nor did the proportions of any of the remaining serotypes in DHF patients differ from their relative proportion in patients overall (Chi-square test, $\mathrm{P}>0.05)$.

\section{Discussion}

Clinical data of pediatric and adult patients in Colombo, Sri Lanka with confirmed dengue fever presenting over a three-year period from 2004 to 2006 were analyzed in this study. The majority of hospitalized patients in the study consisted of children and young adults. Children and adults presented with some complaints that were unique to each age group. Flushing, vomiting and abdominal pain were most common in children whereas arthralgia, myalgia and headache were the most frequent complaints in adults. Thrombocytopenia was more common in adults compared with children. Finally, three of the four serotypes were isolated from pediatric patients over the study period and all four serotypes were isolated from adult patients. 
The median and mean ages for hospitalized pediatric patients in this study were 3 yrs old and 4.8 yrs old respectively, while the estimated median age for all 0-12 yr olds in Colombo is between 5 and 9 yrs old. ${ }^{14}$ In comparison, the patient population in the study was younger than similar studies from South Asia. ${ }^{15,18,19}$ Malavige and colleagues ${ }^{15}$ found a mean age of 7.9 yrs in their study of 104 serologically confirmed hospitalized patients aged 0-12 yrs which is markedly older than patients seen in the current study. The difference between the 2 studies may reflect differences in the particular hospital populations or in testing biases between the two studies. Our study was limited to PCR positive patients at three private hospitals (Apollo, Nawaloka and Durdans Hospitals) from 2003 to 2006 whereas the Malavige study looked at serologically confirmed hospitalized patients at a large public hospital (Lady Ridgeway Hospital) from April to July, 2004. Two other pediatric studies in India found mean ages of $6.5^{18}$ and $6.8^{19} \mathrm{yrs}$ old, similar to the value reported by Malavige et al. Mean age of hospitalized children may also reflect overall background immunity in a population. Changes up or down in the mean age of DENV infection in a given study population suggests changes in background immunity and intensity of virus transmission. The younger the mean age of infection in an endemic area the more intense transmission is likely to have been, as older children are more likely to already be DENV immune. While we cannot confirm that differences in transmission intensity and background immunity explain the mean age differences between our study and the previous studies from South Asia, it is a plausible explanation.

In contrast, the adult demographic data obtained from the present study are similar to previous Sri Lankan studies. While historically, dengue has been primarily a pediatric disease in endemic countries, Sri Lanka has seen many adults present with clinically significant disease requiring hospitalization. ${ }^{8}$ Two previous Sri Lankan studies ${ }^{16,17}$ also reported younger adult patients from among serologically confirmed hospital patients in Sri Lanka, with mean ages of 26.6 and 28 respectively, similar to the mean age of adult patients in the current study.

Gender was a risk factor for dengue infection in both pediatric and adult patients, with males being more likely to be diagnosed with dengue than females (OR 1.36, confidence intervals 1.01, $1.82, \mathrm{P}<0.05)$ ). Several other investigators in the region have reported similar differences in the numbers of male and female patients. ${ }^{18,21,22}$ The most likely explanation is that exposure to the vector in males and females are different. It is also possible, though less likely, that clinical and diagnostic testing biases - clinicians are more likely to suspect dengue in a male patient and hence diagnosis it more often in males - contributed to the observed difference.

This study found that dengue disease presents uniquely in children and adults, consistent with previously published pediatric and adult dengue case reviews. Malavige et al. ${ }^{16}$ identified flushed appearance, vomiting and headache as by far the most frequent findings among hospitalized children in Colombo, while reports from hospitalized pediatric patients in India ${ }^{19}$ noted headache and vomiting to be the most frequent complaint with no mention of a flushed appearance. The current study found vomiting to be the most common complaint at $49 \%$ followed closely by flushing at $48 \%$. Headache in children appeared to be uncommon (14\%). Myalgia/arthralgia were noted in only $8 \%$ of patients in the present study while Kumar et al reported the complaint in $29 \%$ of patients ${ }^{18}$. Malavige and Narayanan ${ }^{24}$ did not comment on myalgia or arthralgia in pediatric patients. The commonest symptoms in adults was myalgia and headache as described 
by Malavige et al. and Kularatne et al. ${ }^{16,17}$ Several other published reports from South Asia, primarily India ${ }^{1,17,26,31}$, also report arthralgia/myalgia and headache as the most frequent complaints among dengue infected hospitalized adults. Whether this difference in pediatric and adult presentation of disease is because of a true difference or is a manifestation of the difficultly in eliciting those particular complaints in children is not clear, though the latter seems a likely explanation.

One notable feature of both the current study and the two earlier Sri Lankan studies ${ }^{16,17}$ was the overall prevalence of abdominal and gastrointestinal complaints, with nearly half $-41.4 \%$ - in the study complaining of vomiting alone and 190 of 292 adults (65.1\%) with at least one gastrointestinal complaint, also described in previous studies. ${ }^{16,17}$ Though not necessarily a classical component of the presentation of dengue, gastrointestinal complaints are part of the clinical syndrome as it presents in Sri Lanka.

The one statistically significant difference between pediatric and adult patients was the proportion of DF patients with thrombocytopenia. We observed thrombocytopenia in $30.9 \%$ of pediatric DF patients and $58.7 \%$ of adult DF patients. Review of other clinical studies in the region finds similar differences between the proportion of thrombocytopenia in pediatric and adult DF patients. Malavige ${ }^{21}$ reported that only $17 \%$ of pediatric DF patients had thrombocytopenia, Kumar ${ }^{15}$ reported thrombocytopenia in $26.4 \%$ of pediatric DF patients during a dengue outbreak in Northern India and Narayanan reported $21.3 \%$ in Chennai, India. ${ }^{24}$ By comparison, Kularatne ${ }^{13}$ found thrombocytopenia in approximately $70 \%$ of adult DF patients and Khan $^{12}$ reported $78 \%$ among DF patients in Saudi Arabia. However, Malavige et al. reported a considerably lower number of adult DF patients with thrombocytopenia, identifying only $24.2 \%$, though still higher that their reported $17 \%$ of pediatric patients. ${ }^{17}$ One significant limitation of this comparison is the timing of blood sample collection. While our samples were collected on day of presentation, which was no later than fever day 4, the platelet nadir which occurs around day 5-7 may not have been reached. In this case, we will have underestimated the true proportion of patients who were thrombocytopenic over the course of their illness.

There were several complications identified in this study. In the pediatric group there were single findings of lower respiratory tract infection, carditis, encephalopathy and two patients with seizures. Among adults, there were single cases of pneumonia and pericardial effusion while 2 patients developed pleural effusions and ascites. No fatalities were reported in the study patient population. Other investigators in South Asia have reported similar complications, including purpura fulminans ${ }^{11}$, carditis $^{14}$, effusions ${ }^{18}$, and encephalopathy ${ }^{20}$, although they are relatively rare and play a minimal role in dengue morbidity and mortality.

The relative abundance of each serotype in Sri Lanka has been previously reported. ${ }^{7,8}$ In this report, as in the past, DENV3 and DENV2 continue to be the dominant infecting serotypes with DENV1 and DENV4 less frequently found in clinical cases. While DENV3 has been previously identified as the likely cause of the emergence of DHF in Sri Lanka, ${ }^{23}$ the current study did not find an increased likelihood of DHF with DENV3 infection. 
Our results, combined with other recent reports of DENV in Sri Lanka, paint an evolving picture of how dengue infection presents in both children and adults in Sri Lanka. While classical DF and DHF predominated, it is clear from our data that the spectrum of disease is broader in terms of symptoms - e.g. abdominal pain - and laboratory parameters - e.g. presence or absence of thrombocytopenia - than classically recognized dengue, and children and adults are likely to present with different constellations of complaints and findings.

\section{References}

1. Agarwal R., Kapoor S., Nagar, R., Misra, A., Tandon, R., Mathur, A. et al. A Clinical Study of the Patients with Dengue Haemorrhagic Fever during the Epidemic of 1996 at Lucknow, India. Southeast Asian J.Trop.Med.Public Health 1999; 30: 735-40. No doi

2. Ahmed, S., Arif, F., Yahya, Y., Rehman, A., Abbas, K., Ashraf, S. et al. Dengue Fever Outbreak in Karachi 2006--a Study of Profile and Outcome of Children Under 15 Years of Age. J.Pak.Med.Assoc. 2008; 58: 4-8. No doi

3. Balmaseda, A., Hammond, S. N., Perez, M. A., Cuadra, R., Solano, S., Rocha, J. et al. Short Report: Assessment of the World Health Organization Scheme for Classification of Dengue Severity in Nicaragua. Am.J.Trop.Med.Hyg. 2005; 73: 1059-62. No doi

4. Dept. of Census and Statistics - Sri Lanka. Census of Population and Housing. Colombo, Sri Lanka: Department of Census and Statistics, 2001.

5. Halstead, S. B. Neutralization and Antibody-Dependent Enhancement of Dengue Viruses. Adv.Virus Res. 2003; 60: 421-67. doi:10.1016/S0065-3527(03)60011-4

6. Hanafusa, S., Chanyasanha, C., Sujirarat, D., Khuankhunsathid, I., Yaguchi, A., Suzuki, T. Clinical Features and Differences between Child and Adult Dengue Infections in Rayong Province, Southeast Thailand. Southeast Asian J.Trop.Med.Public Health 2008; 39: 252-9. No doi

7. Harris, E., Videa, E., Perez, L., Sandoval, E., Tellez, Y., Perez, M. L. et al. Clinical, Epidemiologic, and Virologic Features of Dengue in the 1998 Epidemic in Nicaragua. Am.J.Trop.Med.Hyg. 2000; 63: 5-11. No doi

8. Kabilan, L., Balasubramanian, S., Keshava, S. M., Satyanarayana, K. The 2001 Dengue Epidemic in Chennai. Indian J.Pediatr. 2005; 72: 919-23. doi:10.1007/BF02731664

9. Kabra, S. K., Jain, Y., Pandey, R. M., Madhulika., Singhal, T., Tripathi, P. et al. Dengue Haemorrhagic Fever in Children in the 1996 Delhi Epidemic. Trans.R.Soc.Trop.Med.Hyg. 1999; 93: 294-8. doi:10.1016/S0035-9203(99)90027-5

10. Kanakaratne, N., Wahala, W. M., Messer, W. B., Tissera, H. A., Shahani, A., Abeysinghe, N. et al. Severe Dengue Epidemics in Sri Lanka, 2003-2006. Emerg.Infect.Dis. 2009; 15: 192-9. doi:10.3201/eid1502.080926

11. Karunatilaka, D. H., De Silva, J. R., Ranatunga, P. K., Gunasekara, T. M., Faizal, M. A., Malavige, G. N. Idiopathic Purpura Fulminans in Dengue Hemorrhagic Fever. Indian J.Med.Sci. 2007; 61: 471-3. doi:10.4103/0019-5359.33712

12. Khan, E., Siddiqui, J., Shakoor, S., Mehraj, V., Jamil, B., Hasan, R. Dengue Outbreak in Karachi, Pakistan, 2006: Experience at a Tertiary Care Center. Trans.R.Soc.Trop.Med.Hyg. 2007; 101: 1114-9. doi:10.1016/j.trstmh.2007.06.016

13. Kularatne, S. A., Gawarammana, I. B., Kumarasiri, P. R. Epidemiology, Clinical Features, Laboratory Investigations and Early Diagnosis of Dengue Fever in Adults: A 
Descriptive Study in Sri Lanka. Southeast Asian J.Trop.Med.Public Health 2005; 36: 686-92. No doi

14. Kularatne, S. A., Pathirage, M. M., Kumarasiri, P. V., Gunasena, S., Mahindawanse, S. I. Cardiac Complications of a Dengue Fever Outbreak in Sri Lanka, 2005.

Trans.R.Soc.Trop.Med.Hyg. 2007; 101: 804-8. doi:10.1016/j.trstmh.2007.02.021

15. Kumar, R., Tripathi, P., Tripathi, S., Kanodia, A., Pant, S., Venkatesh, V. Prevalence and Clinical Differentiation of Dengue Fever in Children in Northern India. Infection 2008; 36 (5) 444-9 doi:10.1007/s15010-008-7172-6

16. Kyle, J. L., Harris, E. Global Spread and Persistence of Dengue. Annu.Rev.Microbiol. 2008; 62: 71-92. doi:10.1146/annurev.micro.62.081307.163005

17. Lee, M. S., Hwang, K. P., Chen, T. C., Lu, P. L., Chen, T. P. Clinical Characteristics of Dengue and Dengue Hemorrhagic Fever in a Medical Center of Southern Taiwan during the 2002 Epidemic. J.Microbiol.Immunol.Infect. 2006; 39: 121-9. No doi

18. Lucas, G. N., Amerasinghe, A., Sriranganathan, S. Dengue Haemorrhagic Fever in Sri Lanka. Indian J.Pediatr. 2000; 67: 503-4. doi:10.1007/BF02760477

19. Mackenzie, J. S., Gubler, D. J., Petersen, L. R. Emerging Flaviviruses: The Spread and Resurgence of Japanese Encephalitis, West Nile and Dengue Viruses. Nat.Med. 2004; 10: S98-109. doi:10.1038/nm1144

20. Malavige, G. N., Ranatunga, P. K., Jayaratne, S. D., Wijesiriwardana, B., Seneviratne, S. L., Karunatilaka, D. H. Dengue Viral Infections as a Cause of Encephalopathy. Indian.J.Med.Microbiol. 2007; 25: 143-5. doi:10.4103/0255-0857.32722

21. Malavige, G. N., Velathanthiri, V. G., Wijewickrama, E. S., Fernando, S., Jayaratne, S. D., Aaskov, J. et al. Patterns of Disease in Sri Lankan Dengue Patients. Arch.Dis.Child. 2006a; 91: 396-400. doi:10.1136/adc.2005.085191

22. Malavige, G. N., Velathanthiri, V. G., Wijewickrama, E. S., Fernando, S., Jayaratne, S. D., Aaskov, J. et al.. Patterns of Disease among Adults Hospitalized with Dengue Infections. QJM 2006b; 99: 299-305. doi:10.1093/qjmed/hcl039

23. Messer, W. B., Gubler, D. J., Harris, E., Sivananthan, K., de Silva, A. M. Emergence and Global Spread of a Dengue Serotype 3, Subtype III Virus. Emerg.Infect.Dis. 2003; 9: 800-9. No doi

24. Narayanan, M., Aravind, M. A., Thilothammal, N., Prema, R., Sargunam, C. S., Ramamurty, N. Dengue Fever Epidemic in Chennai--a Study of Clinical Profile and Outcome. Indian Pediatr. 2002; 39: 1027-33. No doi

25. Premaratna, R., Pathmeswaran, A., Amarasekara, N. D., Motha, M. B., Perera, K. V., de Silva, H. J. A Clinical Guide for Early Detection of Dengue Fever and Timing of Investigations to Detect Patients Likely to Develop Complications. Trans.R.Soc.Trop.Med.Hyg. 2008; 103(2): 127-31 doi:10.1016/j.trstmh.2008.07.024

26. Rai, S., Chakravarti, A., Matlani, M., Bhalla, P., Aggarwal, V., Singh, N. et al. ClinicoLaboratory Findings of Patients during Dengue Outbreak from a Tertiary Care Hospital in Delhi. Trop.Doct. 2008; 38: 175-7. doi:10.1258/td.2007.070229

27. Rico-Hesse, R. Microevolution and Virulence of Dengue Viruses. Adv.Virus Res. 2003; 59: 315-41. doi:10.1016/S0065-3527(03)59009-1

28. Rigau-Perez, J. G., Bonilla, G. L. An Evaluation of Modified Case Definitions for the Detection of Dengue Hemorrhagic Fever. Puerto Rico Association of Epidemiologists. P.R.Health Sci.J. 1999; 18: 347-52. No doi 
29. Rothman, A. L. Immunology and Immunopathogenesis of Dengue Disease. Adv.Virus Res. 2003; 60: 397-419. doi:10.1016/S0065-3527(03)60010-2

30. SAS Institute Inc. JMP. Vol. 7. Cary, NC:, 1989-2007.

31. Singh, N. P., Jhamb, R., Agarwal, S. K., Gaiha, M., Dewan, R., Daga, M. K. et al. The 2003 Outbreak of Dengue Fever in Delhi, India. Southeast Asian J.Trop.Med.Public Health 2005a; 36: 1174-8. No doi 